\title{
OBSERVATION OF NONLINEAR BEHAVIOR OF LOCALIZED SPACE-CHARGE WAVES IN SPACE-CHARGE DOMINATED ELECTRON BEAMS*
}

\author{
J. G. Wang ${ }^{\text {a }}$, Y. Zou, H. Suk ${ }^{\mathrm{b}}$, and M. Reiser \\ Institute for Plasma Research, University of Maryland, College Park, MD 20742
}

\begin{abstract}
We present an experimental observation of the abnormal growth of localized nonlinear fast space-charge waves in space-charge dominated electron beams passing through a dissipative channel. High-perveance electron beams with localized perturbations are launched from a gridded electron gun and transported through a short resistive-wall channel consisting of a resistive-film coated glass tube inside a long solenoid providing uniform focusing. The energy width of the space-charge waves developed from the perturbations is measured on both ends of the channel. The experiments have shown that the localized fast spacecharge wave appears to grow under nonlinear perturbations; in the linear regime, as expected, the energy width of slow waves increases, while the energy width of fast waves decreases when they pass through the resistive channel.
\end{abstract}

\section{INTRODUCTION}

The longitudinal instability of charged particle beams, caused by the growth of slow space-charge waves due to interaction with a dissipative wall, is an important issue in particle accelerators, microwave generators, and plasmas. We have been studying this instability in space-charge dominated electron beams by perturbing the beams with localized perturbations and transporting them in a resistive environment. In the previous studies, linear waves were employed and the experiment demonstrated the growth of localized slow space-charge waves and the decay of fast space-charge waves. The growth/decay rates of the slow/fast waves were measured and the experiment was compared with theory [1]. In this paper, we report a unexpected observation that the energy width of localized fast space-charge waves appears to grow in a resistive channel if the perturbations are highly nonlinear.

\section{EXPERIMENTAL SETUP AND METHOD}

The experimental setup, as shown in Fig. 1, consists of a short-pulse electron beam injector, a resistive-wall channel, and diagnostics. The injector [2] contains a gridded electron gun, which can produce desired beam parameters with localized perturbations. The key component of the resistive-wall channel is a glass tube of

\footnotetext{
"Work supported by the U.S. Department of Energy.
}

${ }^{a}$ Email: jgwang@bnl.gov about $1 \mathrm{~m}$ in length and $3.81 \mathrm{~cm}$ in inner diameter. The inner surface of the glass tube is coated with Indium-TinOxide (ITO). The total resistance of the tube employed in the experiments is $5.44 \mathrm{k} \Omega$, which corresponds to 0.673 $\mathrm{k} \Omega$ per square. The beam is focused by a $1.4-\mathrm{m}$ long uniform solenoid coaxial to the resistive-wall tube. Three short solenoidal lenses are employed in the injector to match the beam into this channel. The diagnostics includes two retarding-field energy analyzers at the entrance and the exit of the resistive tube to measure the energy of beam particles and space-charge waves. Typical beam parameters are 2-8 keV in energy, 20-100 $\mathrm{mA}$ in current, and about $100 \mathrm{~ns}$ in the pulse duration.

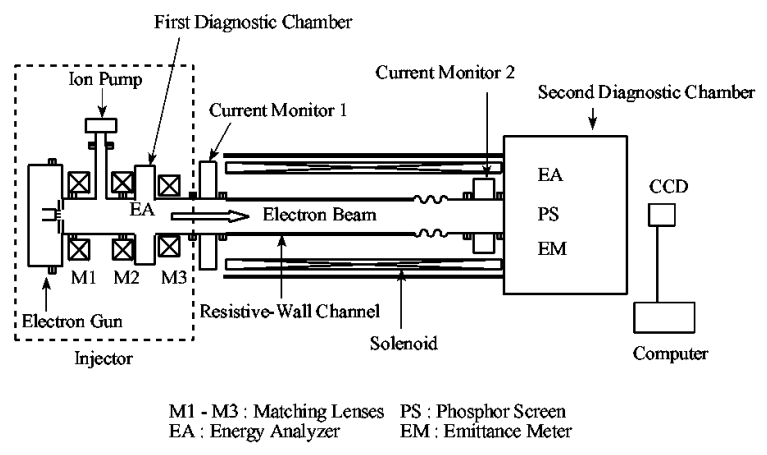

Fig. 1 Setup of resistive-wall instability experiment.

In the experiment, electron beams with localized perturbations are generated in the gridded electron gun and transported through the resistive-wall channel. Single slow or fast waves can be developed on these beam pulses by applying the technique described in a previous letter [3]. The energy width of space-charge waves is measured and compared at the both ends of the resistive-wall channel. This is done as the follows: by increasing the retarding voltage in the energy analyzers, the beams and space-charge waves can be gradually suppressed in the analyzer output. In this way, the energy levels of the beam and the energy width of space-charge waves can be determined. A typical measurement with the energy analyzers is shown in Fig. 2. The beam is suppressed at a retarding high voltage of $3.595 \mathrm{kV}$. The remaining signal on the top trace is a fast wave in which the particles have a higher energy than the beam average energy. When the retarding high voltage further increases, the space-charge wave signal decreases and eventually disappears at 3.608 $\mathrm{kV}$. This results in an energy width of $13 \mathrm{eV}$ for the fast wave. 


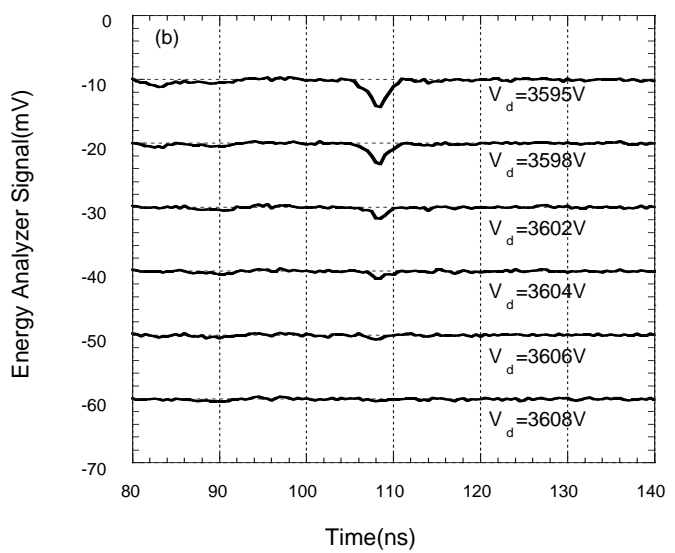

Fig. 2 Fast wave signals from an energy analyzer at different retarding high voltage.

\section{EXPERIMENTAL OBSERVATIONS}

The measurement with the two energy analyzers for a slow wave on a beam of about $2.5 \mathrm{keV}$ and $39.2 \mathrm{~mA}$ is shown in Fig. 3. Here the relative amplitude of the wave from the two energy analyzers is plotted against the difference between the retarding voltage and beam voltage. The energy width of the slow wave increases from $28 \mathrm{eV}$ to $43 \mathrm{eV}$ due to the instability, corresponding to a spatial growth rate of $0.45 / \mathrm{m}$. Another measurement for a fast wave is performed at a beam energy of $3.595 \mathrm{kV}$ and a beam current of $19.8 \mathrm{~mA}$. The result is shown in Fig. 4. The energy width of the fast wave decreases from $21 \mathrm{eV}$ to $13 \mathrm{eV}$, giving a spatial decay rate of $-0.50 / \mathrm{m}$.

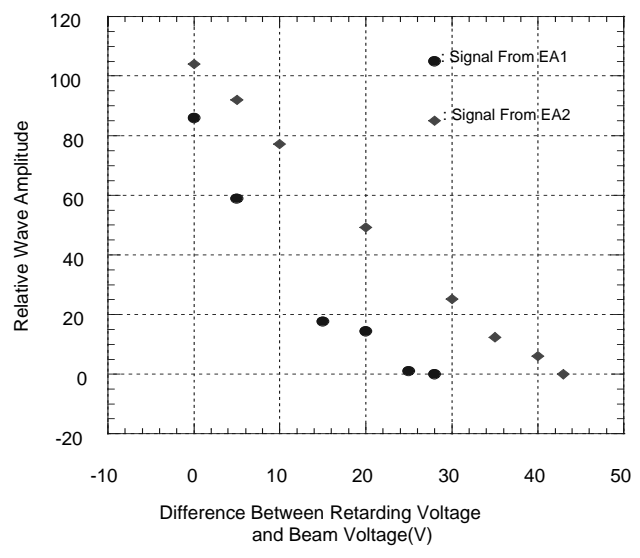

Fig. 3 Relative amplitude of a slow space-charge wave vs. the difference between the retarding voltage and beam voltage, where EA1 and EA2 are the energy analyzer before and after the resistive wall, respectively.

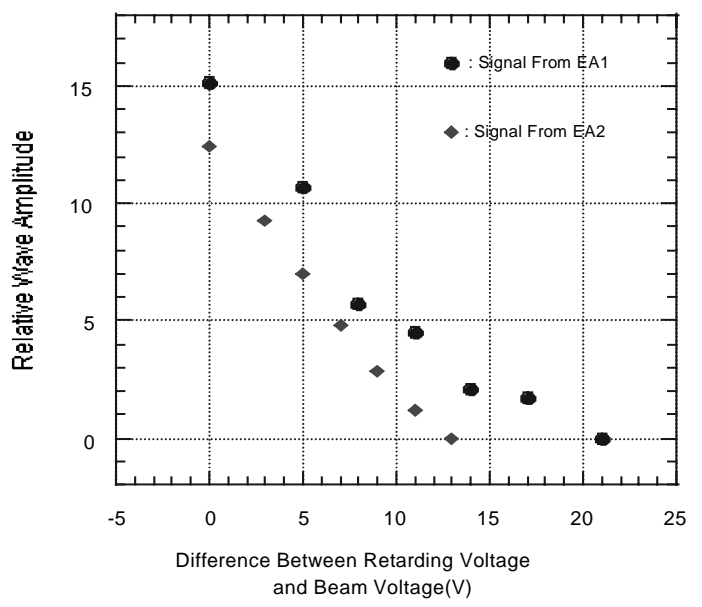

Fig. 4 Energy width of a fast wave from EA1 and EA2.

Measurements with the energy analyzers are also performed in the nonlinear regime where a rather large line-charge density perturbation is introduced on the beams. This is done by introducing a strong voltage perturbation on the grid-cathode pulse of the electron gun, that results in large line-charge density and current perturbation on the beam. Figure 5 shows such a case, where it can be seen that a beam current signal modulated with a large localized space-charge wave, measured before the resistive wall. The ratio of the wave amplitude over the average beam current is nearly $40 \%$, a highly nonlinear case ! Under such nonlinear perturbations, the energy width of a localized slow wave always increases when the wave passes through the resistive channel. This is shown in Fig. 6, where the growth rate of slow waves vs. the perturbation strength is plotted.

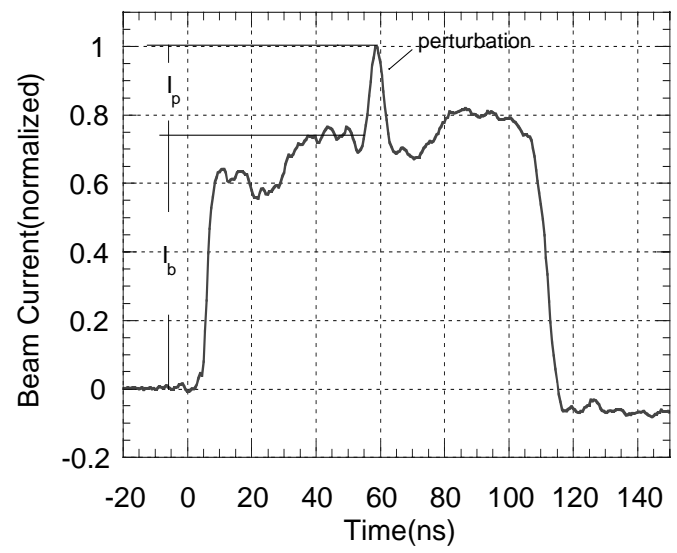

Fig. 5 Beam current signal with a highly nonlinear spacecharge wave. 


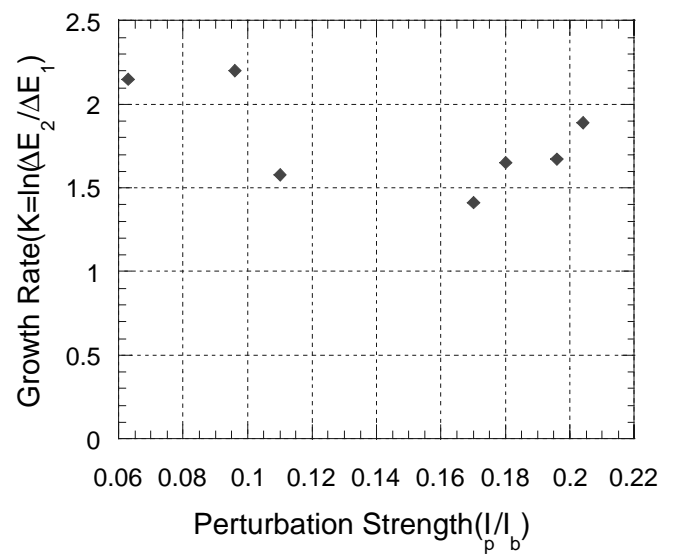

Fig. 6 Growth rates vs. perturbation strength for slow waves.

Nevertheless, the results for localized fast waves are totally unexpected and counter-intuitive. The energy width of the fast waves appears to increase under nonlinear perturbations in the resistive wall channel. This is shown in Fig. 7. Measurements have been performed for fast waves under different perturbation strength. In the linear regime, the fast wave decays, as expected. When the perturbation is strong enough, a transition from decay to growth takes place. The growth rate eventually levels off for the strongest perturbations in the experiments.

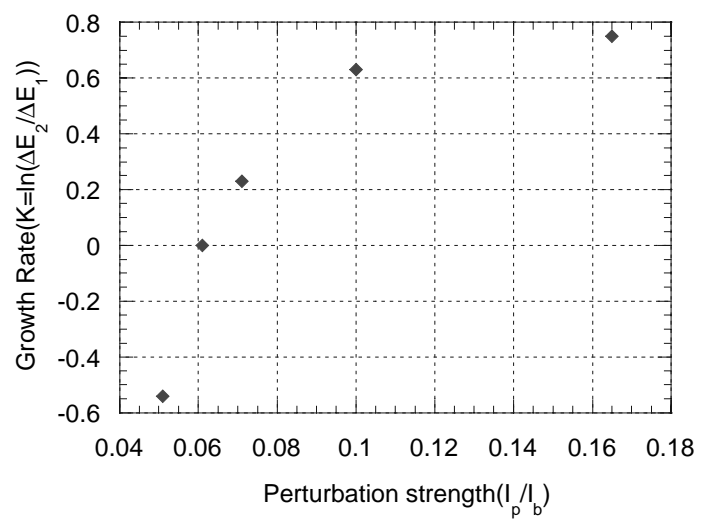

Fig. 7 Growth rates vs. perturbation strength for fast waves in a resistive-wall channel.

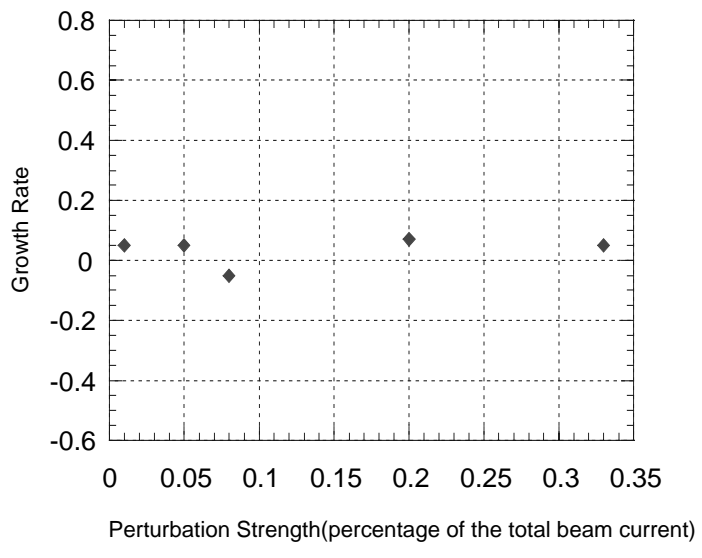

Fig. 8. Growth rates vs. perturbation strength for fast waves in a conducting wall pipe.

We are so far not able to interpret this unexpected phenomenon by the nonlinear dynamics, including the solitary waves, explosive instability, etc. In order to eliminate possible errors in the diagnostics, the resistive wall is replaced by a conducting tube and any other conditions remain the same. In this case, no growth or decay is observed for the fast wave, as expected. The results are plotted in Fig. 8.

\section{REFERENCES}

${ }^{a}$ Present Address: ORNL and Visiting at BNL.

${ }^{\mathrm{b}}$ Present Address: Dept. of Physics, UCLA.

[1] J. G. Wang, H. Suk, and M. Reiser, Phys. Rev. Lett. 79 (6), 1042 (1997).

[2] J. G. Wang, D. X. Wang, and M. Reiser, Nucl. Instr. \& Meth. in Phys. Res. A 316, 112 (1992).

[3] J. G. Wang, D. X. Wang, and M. Reiser, Phys. Rev. Lett., 71(12), 1836 (1993). 